\title{
Geographic information system (GIS)-based image analysis for assessing growth of Physarum polycephalum on a solid medium
}

\author{
Hanh T. M. Tran ${ }^{*}$, Steven L. Stephenson ${ }^{2}$ and Jason A. Tullis ${ }^{3}$
}

\begin{abstract}
Background: The conventional method used to assess growth of the plasmodium of the slime mold Physarum polycephalum in solid culture is to measure the extent of plasmodial expansion from the point of inoculation by using a ruler. However, plasmodial growth is usually rather irregular, so the values obtained are not especially accurate. Similar challenges exist in quantification of the growth of a fungal mycelium.
\end{abstract}

Results: In this paper, we describe a method that uses geographic information system software to obtain highly accurate estimates of plasmodial growth over time. This approach calculates plasmodial area from images obtained at particular intervals following inoculation. In addition, the correlation between plasmodial area and its dry cell weight value was determined. The correlation could be used for biomass estimation without the need of having to terminate the cultures in question.

Conclusion: The method described herein is simple but effective and could also be used for growth measurements of other microorganisms such as fungi on solid media.

Keywords: Myxomycetes, Image, GIS, Plasmodium, Solid culture

\section{Background}

Physarum polycephalum Schwein. is a member of the order Physarales of the class Myxomycetes, a group of fungus-like eukaryotic organisms commonly known as slime molds. Like all other members of this group, the life cycle of $P$. polycephalum is characterized by a distinctive multinucleate trophic (feeding) stage called a plasmodium. The plasmodium of $P$. polycephalum occurs on decaying plant material and the fruiting bodies of wooddecaying fungi in nature and has a bright yellow color [1]. The rapid rate of growth, the absence of cell walls and the ease with which the plasmodium of $P$. polycephalum can be cultured have caused it to be widely used in cell biology and behavioral research.

Research that involves a determination of the growth of P. polycephalum growth on a solid medium is commonly

\footnotetext{
*Correspondence: ttmhanh@hcmiu.edu.vn

${ }^{1}$ School of Biotechnology, Ho Chi Minh International University, Ho Chi Minh City 70000, Vietnam

Full list of author information is available at the end of the article
}

carried out by measuring the extent of plasmodial expansion from the point of inoculation by using a ruler [2-6]. Although this is a convenient method that requires very little effort, plasmodial growth is often irregular in shape, which makes any determination of actual size rather challenging. Less commonly, the plasmodium in a particular culture is collected and dried for determination of plasmodial growth by weighing the dry material on a small balance. The value obtained in such a manner is referred to the dry cell weight (DCW) of the culture. This method is more accurate than a measurement taken with a ruler, but the cultures must be terminated in order to collect the total biomass of the plasmodium [7].

As a general rule, geographic information system (GIS) software has been used in science, engineering, and business applications at spatial scales linked to common methods of locational observation (e.g., global positioning systems as well as airborne and satellite-based imaging). However, extension of GIS to finer scales has produced innovations such as 3D topographic analysis of 
primate teeth (e.g., Zuccotti et al. [8]). In a rare microbiological application, Yang et al. [9] used GIS for measuring fungal growth. The mycelial cultures of the fungi being cultured were photographed and fungal growth was determined on the basis of the proportion of the pixel count of the mycelium divided by the sum of the pixel count of the mycelium and substrate. Our comparable approach, also based on pixel counts, represents a new application of GIS to study the growth of a plasmodium. The relatively precise aerial estimates (i.e., when compared with the use of a ruler) are facilitated by (a) digital camera capture of fine spatial resolution 2D images of plasmodia and (b) GIS-based extraction of areal extents of visually unique patterns in the imagery.

Originally based on the work of Ball and Hall [10], ISODATA clustering is commonly available in GIS software and used to enable classification of (or categorical labeling of pixels in) imagery acquired from various cameras and other types of sensors. ISODATA is well suited to cluster (or group) pixels of various colors found in a typical digital camera-captured image of a Petri dish. For example, the various intensities of the yellow color of the plasmodium, the clear raw substrate, the plastic walls of the dish, and the background materials are each candidates for a cluster, depending on the parameterization of ISODATA. The proportion of yellow plasmodium pixels, relative to the total number of pixels in the Petri dish, can be multiplied by the area of the Petri dish to obtain a systematic area of the plasmodium at the time the photo is acquired. Moreover, this approach is especially useful for any project with the goal of tracking the growth of a particular plasmodium over time or for comparing plasmodial growth between different cultures.

The objectives of the project reported herein were first to evaluate the feasibility of using GIS software as a new approach for measuring plasmodial growth and then to determine the correlation (if any) between the pixel areas of plasmodia and their dry cell weight (DCW) increments.

\section{Results and discussion}

\section{Images of plasmodial growth profile}

Physarum polycephalum was grown in nutrient agar medium culture plates. Each plate contained $25 \mathrm{~mL}$ of medium. Inoculum was added to the center of the plate. It can be noticed that the plasmodial extensions were irregular and when the plasmodia migrated away from the points of inoculation, they formed slime tracks, which were colorless. This can be observed from the 36 and $48 \mathrm{~h}$ images (Fig. 1).

After $48 \mathrm{~h}$, the plasmodia began clumping together and formed thicker biomass (image not shown). More discussion about this phenomenon is provided below.

\section{GIS analysis of plasmodial images}

Eight plasmodial cultures were prepared using the same inoculum size and medium composition as described above. Every $12 \mathrm{~h}$, images of the cultures were obtained and plasmodium was collected from two randomly selected plates for later comparison of growth area and DCW analysis. The original images of the plasmodial culture used for GIS analysis were taken using a Canon EOS $100 \mathrm{D}$ camera. All of the images had the same resolution $(1920 \times 1280$ pixels $)$ and were saved as JPEG files.

The plasmodial images were analyzed using Esri's ArcGIS 10.2 for Desktop GIS software with the Spatial Analyst (image analysis) extension enabled. An iterator (for automated looping through plasmodium images) was set up using a graphic block programming capability (ModelBuilder) native to ArcGIS. When executed, the iterator transformed each original JPEG plasmodium image into a grayscale image, with each brightness value representing a unique cluster of similar pixels. Through ISODATA, a signature of each cluster (or unique group of pixels in the plasmodium photo) was determined and reported as (a) mean blue, green, and red brightness values, and (b) a three-band variance-covariance matrix. Configured to search for 100 unique clusters, ISODATA used an iterative process to calculate up to 100 of these unique signatures [10].

With the ISODATA signatures used for reference, a Maximum Likelihood Classifier (MLC) was applied to sort each pixel of the image into a cluster. This produced a grayscale image showing the patterns for the various components (e.g., Petri dish, plasmodium, etc.) but with each brightness value representing a unique cluster or color subcomponent of the image (Fig. 2). Using the diameter of the Petri dish in pixels and a spreadsheet, the pixel counts for a selection of plasmodium clusters were obtained and converted to a value for area.

While comparable to the technique used by Yang et al. [7] in terms of counting target pixels, this technique relies upon selection of appropriate target (plasmodium) clusters instead of extraction of pixels based on a blue channel threshold. This technique also eliminates the need for masking out background components of the image, since these simply become clusters that will not be selected.

Many software tools can be adapted to achieve the same image processing results described above, and a number of studies have demonstrated successful image processing approaches for observing spatial and temporal patterns of plasmodia [11-14]. The GIS-based image analysis approach offers an expanding array of tools and intuitive interfaces, workflows history (provenance), and excellent documentation influenced by the current proliferation of multidisciplinary image-producing technologies. 

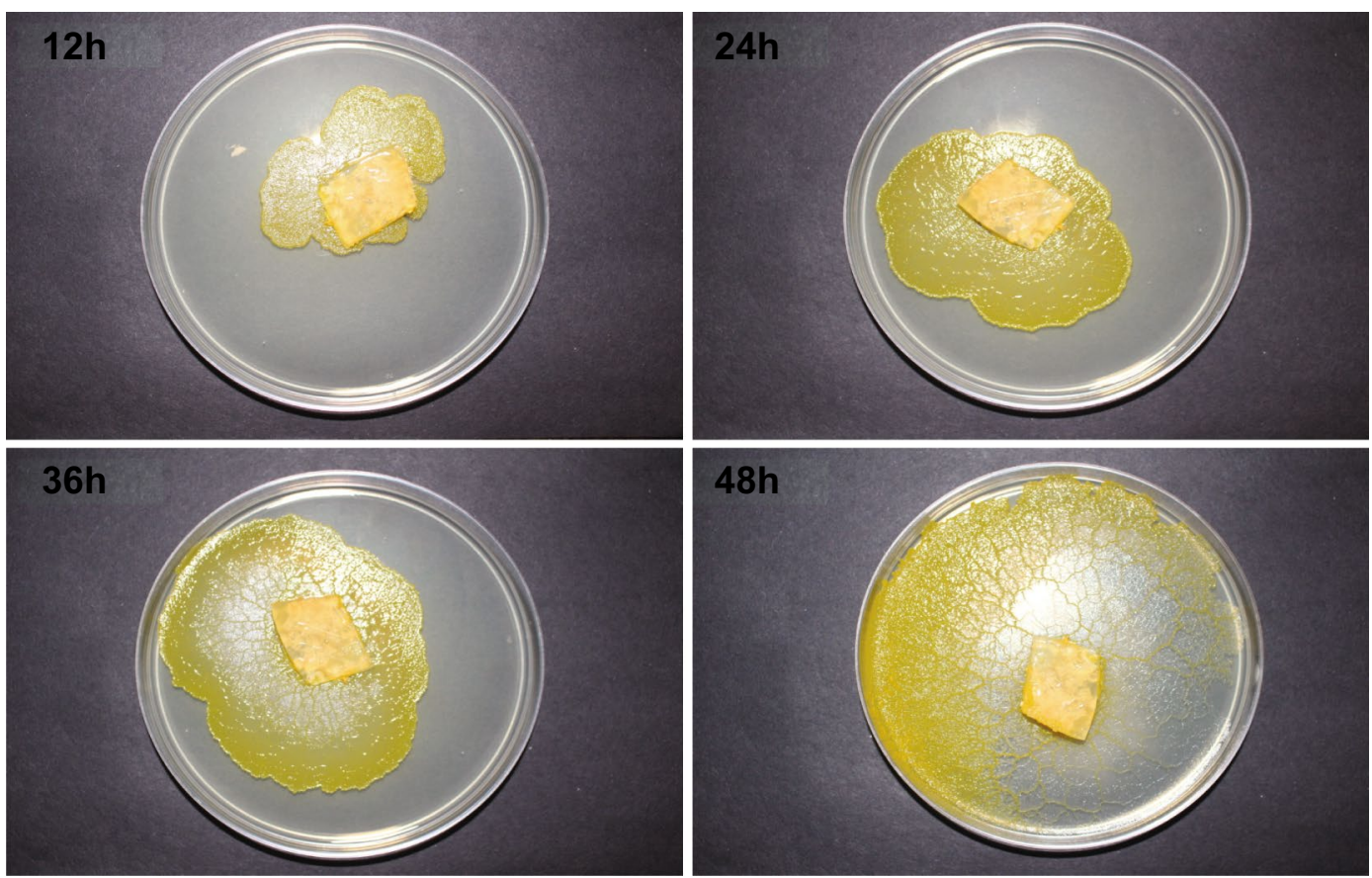

Fig. 1 Images of P. polycephalum plasmodia taken at different times after inoculation from the same starting culture

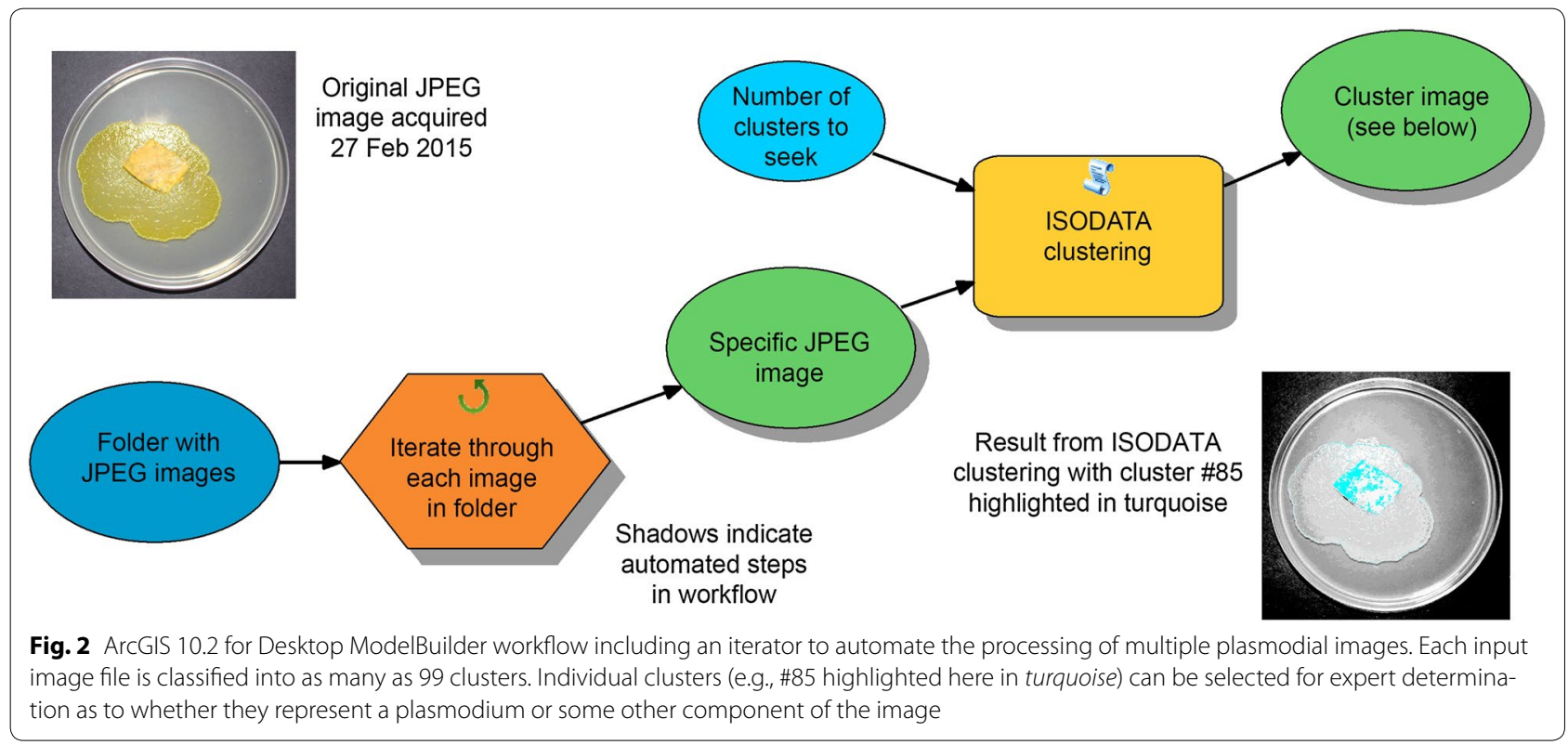

\section{Pixel area and DCW profiles of plasmodial cultures}

As indicated in Fig. 3, both pixel area and DCW of the culture increased exponentially over time. It should be noted that after $48 \mathrm{~h}$, the plasmodium began clumping together and formed areas with a thicker biomass. The DCWs still kept increasing exponentially, but pixel area of the corresponding plasmodium did not follow the same pattern; however, it was still significantly higher than the previous value. Presumably, to avoid plasmodial cluster formation, larger containers would need to be used. However, even with cluster formation it should be possible to extract more precise geometric estimates of 


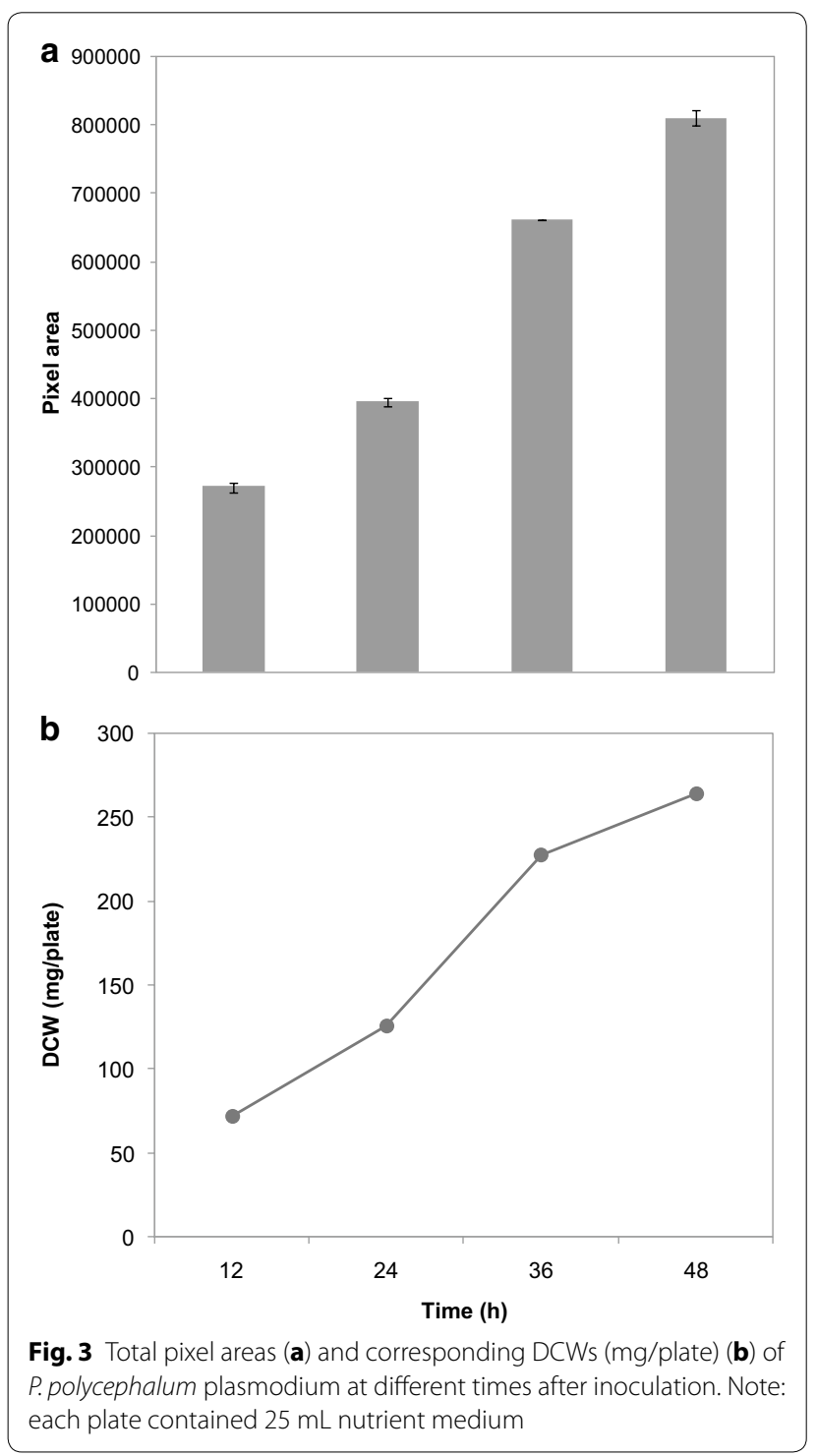

plasmodial growth from 2D digital photographs and GIS. For example, varying degrees of plasmodial thickness will naturally register as different shades of yellow, and therefore may be grouped into different ISODATA clusters. Using an average estimate of plasmodial thickness for given shades of yellow, it should be possible to estimate plasmodial volume from the respective $2 \mathrm{D}$ areas. Future research should examine this approach to determine if improved correlations between GIS-assisted and DCWbased measurements.

\section{Correlation between plasmodial culture area and DCW}

The relationship between plasmodial culture area and DCW was calculated as

$$
y=2757.1 x+59126\{70<x<270\}
$$

where $y$ is pixel area and $x$ is DCW (mg) (Fig. 4). With $\mathrm{R}^{2}$ at 0.99 (over the range observed from approximately 70-270 mg DCW), this relationship is very promising.

As mentioned previously, however, a high degree of accuracy with respect to data on pixel area would be obtained only if the plasmodium does not form clusters.

Determining the correlation between the pixel area of a plasmodium and its DCW provides researchers an additional option for assessing plasmodial growth and the actual product (biomass) being produced over time without terminating the cultures. For instance, the lipid content of the $P$. polycephalum plasmodium was found to be approximately $10 \%$ of the DCW [7]. In the most commonly used method to determine the lipid amount of a culture, the plasmodium is collected and DCW is determined to derive an estimation of the lipid amount. However, in the method described herein, which uses GIS software, DCW can be calculated from pixel area and the amount of lipid determined accordingly.

\section{Conclusions}

A new approach for myxomycete plasmodial growth measurement was developed using GIS software. This approach allows a more accurate assessment of the growth of the plasmodium over time than can be obtained with the use of traditional methods. Moreover, the correlation between pixel area of a plasmodial image and its DCW can be used for biomass estimation with a high degree of accuracy.

\section{Methods}

\section{Physarum polycephalum}

The strain of $P$. polycephalum used in the present study was obtained as a sclerotium from Carolina Biological Supply Company (Burlington, North Carolina).

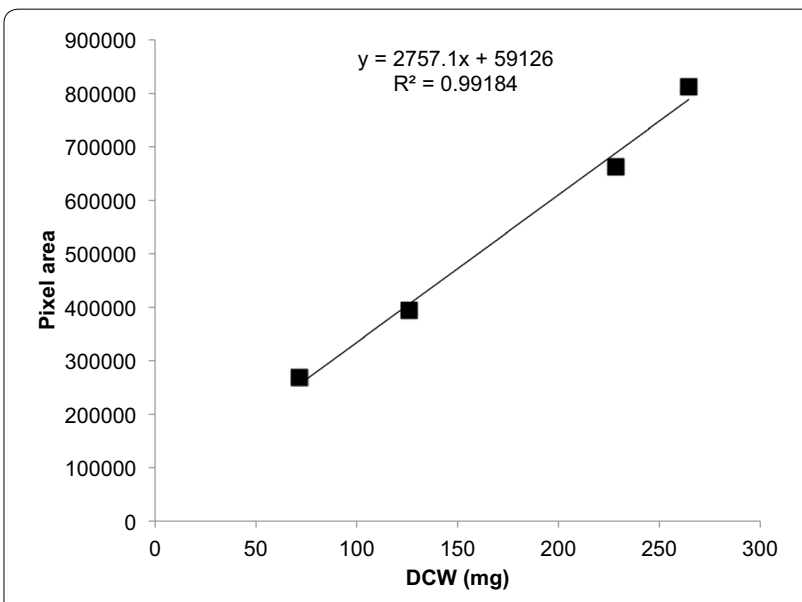

Fig. 4 Correlation between pixel area and DCW of the culture 


\section{Medium used}

The medium used for activation of the sclerotium was water agar $(1.0 \mathrm{~L}$ of water agar containing $20.0 \mathrm{~g}$ of agar and $1000 \mathrm{~mL}$ of distilled water), whereas the medium used for plasmodial growth was nutrient agar $(1.0 \mathrm{~L}$ of the nutrient agar containing $100 \mathrm{~mL}$ of a basal salt solution, $5.0 \mathrm{~g}$ of glucose [Difco], $2.5 \mathrm{~g}$ of yeast extract [Difco], $20.0 \mathrm{~g}$ of agar, and $900 \mathrm{~mL}$ of distilled water adjusted to $\mathrm{pH}$ 5.5). The basal salt solution contained $29.78 \mathrm{~g}$ of citric acid, $33.10 \mathrm{~g}$ of $\mathrm{K}_{2} \mathrm{HPO}_{4}, 2.50 \mathrm{~g}$ of $\mathrm{NaCl}, 1.00 \mathrm{~g}$ of $\mathrm{MgSO}_{4} \cdot 7 \mathrm{H} 2 \mathrm{O}, 0.50 \mathrm{~g}$ of $\mathrm{CaCl}_{2} \cdot 2 \mathrm{H}_{2} \mathrm{O}$, and $1000 \mathrm{~mL}$ distilled water.

\section{Plasmodial activation}

The plasmodium of $P$. polycephalum was activated by placing the sclerotium on the surface of a water agar plate (10 cm diameter). Once activated, sterile oat flakes were added on the actively growing plasmodium and the latter incubated in the dark for 1 day. A plug of agar $\left(\mathrm{ca} 2 \mathrm{~cm}^{2}\right)$ bearing a portion of the active plasmodium growing in association with the oat flakes was used as an inoculum and transferred to larger plates (14 cm in diameter) containing different types of media. Plates were incubated in the dark at room temperature (ca. $22-23{ }^{\circ} \mathrm{C}$ ).

\section{Acquiring images of cultures}

A digital image of each culture was obtained every $12 \mathrm{~h}$. In order to maintain the same distance, lightning and size/aspect in all of the images, the camera used to take the images was attached to a small tripod and the latter remained untouched during the entire period the experiment was carried out except when each new image was acquired. Black paper was used as the background to achieve maximum contrast with the color of the culture dish and plasmodium. The position at which the cultures were placed was predetermined and marked on the paper. It should be noted here that to avoid light reflection, paper with a rough surface was selected instead of one with a shiny/smooth surface. In addition, to minimize color bias, culture information was recorded separately on a removable label and the latter was not included in the image obtained of the culture.

\section{Determination of dry cell weight}

Fresh biomass of $P$. polycephalum was collected from each culture and lyophilized (Labconco, freeze-zone 6) to a constant weight and weighed on an analytical balance (AB104-S, Switzerland). This value represented the dry cell weight (DCW).

\section{Experimental statistics}

All the experiments were carried out in duplicate. The figures included in this paper are derived from the mean values ( \pm standard errors) obtained from both sets of cultures.

\section{Authors' contributions}

HT carried out the culturing experiments, acquired the images of the cultures and partially analyzed the data, SS helped with the culturing techniques and prepared the manuscript, whereas JT analyzed the images. All authors read and approved the final manuscript.

\section{Author details}

${ }^{1}$ School of Biotechnology, Ho Chi Minh International University, Ho Chi Minh City 70000, Vietnam. ${ }^{2}$ Department of Biological Sciences, University of Arkansas, Fayetteville, AR 72701, USA. ${ }^{3}$ Department of Geosciences, University of Arkansas, Fayetteville, AR 72701, USA.

\section{Acknowledgements}

This project was funded in part by a Provost's Collaborative Research Grant from the University of Arkansas.

\section{Competing interests}

The authors declare that they have no competing interests with respect to any aspect of this manuscript or the project reported in the manuscript.

Received: 31 August 2015 Accepted: 11 November 2015

Published online: 19 November 2015

\section{References}

1. Stephenson SL, Stempen H. Myxomycetes: a handbook of slime molds. Oregon: Timber Press; 1994.

2. Knowles CJD, Carlile JM. Growth and migration of plasmodia of the myxomycete Physarum polycephalum: the effect of carbohydrates, including agar. J Gen Microbiol. 1978;108:9-15.

3. Clark JD, Landolt JC, Stephenson SL. The effects of dictyostelids on the formation and maturation of myxomycete plasmodia. Mycologia. 2002;94:933-8.

4. Latty T, Beekman M. Food quality affects search strategy in the acellular slime mould, Physarum polycephalum. Behav Ecol Sociobiol. 2009;20:1160-7.

5. Terry CL, Stefanski MJ, Eisemann AB. Effects of calcium on growth of Physarum polycephalum. Bios. 2009;80:159-63.

6. Reid RC, Beekman M, Latty T, Dussutour A. Amoeboid organism uses extracellular secretions to make smart foraging decisions. Behav Ecol Sociobiol. 2013;24:812-8.

7. Tran HTM, Stephenson SL, Chen J, Pollock ED, Goggin FL. Evaluating the potential use of myxomycetes as a source of lipids for biodiesel production. Bioresour Technol. 2012;123:386-9.

8. Zuccotti LF, Williamson MD, Limp WF, Ungar PS. Technical note: modeling primate occlusal topography using geographic information systems technology. Am J Phys Anthropol. 1998;107(1):137-42.

9. Yang J, Jingyin Z, Qian G, Yunsheng W, Wang R. Data acquisition method for measuring mycelium growth of microorganism with GIS. In: Li D, Liu Y, Chen Y, editors. Computer and computing technologies in agriculture IV. Nanchang: Springer; 2011. p. 374-80.

10. Ball, GH, Hall, DJ. ISODATA, a novel method of data analysis and pattern classification. Report Number AD0699616. Menlo Park, CA: Stanford Research Institute; 1965.

11. Baumgarten W, Ueda T, Hauser JBM. Plasmodial vein networks of the slime mold Physarum polycephalum form regular graphs. Phys Rev E. 2010;82(046113):12

12. Ito M, Okamoto R, Takamatsu A. Characterization of adaptation by morphology in a planar biological network of plasmodial slime mold. JPSJ. 2011;80:074801.

13. Alim K, Amselem G, Peaudecerf F, Brenner PM, Pringle A. Random network peristalsis in Physarum polycephalum organizes fluid flows across an individual. PNAS. 2013;110:13306.

14. Vidal-Diez de Ulzurrun G, Baetens JM, Van den Bulcke J, Lopez-Molina C, De Windt I, De Baets B. Automated image-based analysis of spatio-temporal fungal dynamics. Fungal Genet Biol. 2015;84:12-25. 